\title{
Oral health and stomatological complications in pediatric cancer patients
}

\author{
Isabel Nemoto Vergara Sasada ${ }^{1}$, Lauro José Gregianin² ${ }^{2}$ and Maria Cristina Munerato*3 \\ ${ }^{1}$ Pediatric and Adolescent Health, Dentistry Unit, Hospital de Clínicas de Porto Alegre, Federal University of Rio Grande do Sul, Brazil \\ ${ }^{2}$ Pediatric and Adolescent Health, Pediatric Dentistry Service, Hospital de Clínicas de Porto Alegre, Federal University of Rio Grande do Sul, Brazil \\ ${ }^{3}$ Stomatology, Stomatology Unit, Hospital de Clínicas de Porto Alegre, and Associated Professor, Faculty of Dentistry, Federal University of Rio Grande do Sul, \\ Brazil
}

\begin{abstract}
Objective: To assess oral health status and its relationship with stomatological complications, oral mucositis (OM) and odontogenic infections (OI), after dental work in pediatric patients with cancer.

Design: Prospective cohort study and analysis of oral health conditions with a 6-month follow-up.

Setting: Dental Unit, Stomatology Unit and Oncology Service, Hospital de Clínicas de Porto Alegre (HCPA), Federal University of Rio Grande do Sul, Brazil.

Participants: Sixty-five pediatric patients with cancer, 36 boys and 29 girls; aged 6 months to 18 years; 839 dental evaluations during oncologic treatment and comparisons between two patients groups - inappropriate oral health and appropriate oral health - considering the risk for stomatological complications manifesting as odontogenic infections and mucositis related to chemotherapy, gender, age group, head and neck radiation therapy and time to onset of complications.

Results: Thirty-three patients had inappropriate oral health. The group with appropriate oral health had lower dental infection rates $(P=0.003)$, and mucositis emerged later, compared with the inappropriate health group $(\mathrm{P}<0.001)$. The data suggest that the group with inappropriate oral health had increased odontogenic infection rates, independently of the chemotherapeutic protocol adopted, when mucositis emerged earlier (when the patient presented hyperemic mucosa and burning sensation). Patients with deciduous teeth (6 months a 6 years) had lower incidence of mucositis (69.2\%). Males had higher incidence of mucositis (80.6\%). In the permanent dentition range (13-18 years) there was a higher incidence of dental infections (41.2\%). Girls were more often associated with dental infections (41.4\%).

Conclusion: Studies on dental work in pediatric cancer patients are very limited. Our research contributed to clarify patient profile and needs. The results will be fundamental for the development of protocols and service strategies directed to this population. Educational measures alone do not reduce the risk of odontogenic infections.
\end{abstract}

Abbreviations: MO: Oral mucositis; OI: odontogenic infections; HCPA: Hospital de Clínicas de Porto Alegre; ALL: acute lymphocytic leukemia; POS-HCPA: Pediatric Oncology Service - Hospital de Clínicas de Porto Alegre; AOH: appropriate oral health; IHO: inappropriate oral health; WHO: World Health Organization; HR: hazard ratio; $\mathrm{OHH}$ : Oral hygiene habits ; DMFT index: decayed, missing, and filled teeth index; VPS: visible plaque score; GBI: gingival bleeding index; LILT: low-intensity laser therapy

\section{Introduction}

Neoplasias affecting children and adolescents represent between $1 \%$ and $3 \%$ of all malignant tumors [1]. In Brazil, 2012 estimates revealed that approximately 11,530 new cancer cases are diagnosed in these age groups [1]. Leukemias, especially acute lymphocytic leukemia (ALL), are the main disease to cause death in 5-year-old children and over [1]. Pediatric tumors have specific characteristics such as short latency periods, greater aggressiveness, and fast growth compared with neoplasias in adult populations [1]. However, pediatric tumors respond more effectively to oncologic treatment and, as a rule, have better prognosis. The kind of oncologic treatment, chemotherapy, radiotherapy and/or surgery varies with tumor type [1].
Despite the considerable progress in cancer treatment strategies, opportunistic infections stand as the second main cause of death amongst pediatric patients. Several risk factors associated with infection worsen morbidity rates, the most important of which are the immunosuppressant effects of cytotoxic chemotherapeutic protocols, extended hospital stays, the use of broad-spectrum antibiotics, apart from the loss of integrity associated with oral mucositis (OM) and with the need for central venous access for the catheter. Although some viral pathogens are involved, bacteria are the main agents responsible for most complications linked with death risk in these patients [2].

It is known that chemotherapy-induced $\mathrm{OM}$ is a self-limiting condition when a secondary source of contamination is not involved (tabela 1). Opportunistic infectious processes may spread across the

Correspondence to: Maria Cristina Munerato, Faculty of Dentistry, Federal University of Rio Grande do Sul, Rua Ramiro Barcelos, 2492, 90035-903 Porto Alegre, RS, Brazil, Tel: 5551 993661978; E-mail- mcmunerato@gmail.com

Key words: stomatitis, chemotherapy, radiotherapy, oral health, pediatric oncology

Received: November 28, 2016; Accepted: December 16, 2016; Published: December 19, 2016 
oral mucosa, interfering in treatment and in compromising survival $[3,4]$.

Odontogenic infections (OI) are broadly defined as infectious processes caused by the bacterial flora that is also responsible for periodontal disease and caries. These processes vary from localized infections that require only odontological treatment to those that spread across oral tissues and adjacent anatomic planes, even to the mediastinum. Situations of such severity demand a more aggressive therapeutic approach, and may pose death risk [5].

Health promotion initiatives, specific protection measures based on oral hygiene protocols, early diagnosis and treatment, minimization of damage, and rehabilitation are among the main efforts in oral health applicable to this patient population [6]. Septic episodes in neutropenic patients after chemotherapy are associated with the oral microbiota and may be attenuated by early dental intervention [7].

In this scenario, the objective of the present study was to evaluate the oral health conditions in children and adolescents in cancer treatment and assess the association between these conditions and $\mathrm{OM}$ and OI during this treatment.

\section{Methods}

This prospective cohort study was carried out between May 2011 and December 2012 with a 6-month follow-up after the oncologic diagnostic. Pediatric patients with cancer aged between 6 months and 18 years attending the Pediatric Oncology Service (POS) of the Hospital de Clínicas de Porto Alegre (HPCA), Porto Alegre, Brazil, were selected by convenience sampling.

Inclusion criteria were age between 6 months and 18 years, cancer diagnosis, signing of a consent form by parents or custodians, and participation in an instruction session to prevent oral diseases. Exclusion criteria were edentulous patients, and refusal by parents or custodians to participate in this study.

Patients were divided into two groups based on clinical dental examination (in some cases, radiological findings were also considered): appropriate oral health patients $(\mathrm{AOH}$, that is, without oral diseases such as caries, gingivitis, and oral infections), and inappropriate oral health patients (IOH, who presented caries, tooth root remains, gingivitis, and oral diseases). The allocation of patients considered the preexistence of these conditions, since these may affect the incidence of stomatological complications after exposure to potentially toxic drugs.

Patients and family members were given preventive and educational dental care. The patients in the $\mathrm{IOH}$ group were submitted to specific dental treatment (tooth extractions, restoration of teeth with caries, treatment of preexistent oral infections with appropriate drugs) until an appropriate level of oral health was achieved. Dental treatment sessions were carried out prior to chemotherapy whenever possible, by the same dental surgeon. Because of the urgency of cancer treatment, $45.4 \%$ of patients received full dental care (elimination of all oral infection foci), while $54.6 \%$ were given partial treatment (treatment of painful dental urgency conditions and tooth abscesses). The osteomyelitis of the jaws and the pericoronitis are two examples of OI.
The oncologic therapeutic protocol was carried out in accordance with the routine practice in the POS-HPCA. The medical drugs used are recommended in the protocols and were divided into two groups, high risk and low risk for OM. The protocol based on high OM risk drugs [8,9] uses the drugs methotrexate, doxorubicin, and cyclophosphamide, which are used to treat osteosarcoma, Ewing's sarcoma, acute lymphocytic leukemia (ALL), and Burkitt's lymphoma; these antitumor agents are part of the treatments, and stomatotoxicity is one of their described adverse effects.

The protocol based on low OM risk was administered to patients with medulloblastoma, Wilms' tumor, glioma, rhabdomyosarcoma, retinoblastoma, histiocytosis, neuroblastoma, testes tumor, ovary tumor, troncocerebral tumor, astrocytoma, Hodgkin's lymphoma, chromic myeloid leukemia, and leiomyosarcoma.

Patients still in hospital and outpatients were given stomatological follow-up every 21 days, which coincided with the chemotherapy cycles along at least six months. OM is classified by WHO based on a 4-stage grading system: grade 0 - no changes; grade 1 - pain/erythema; grade 2 - erythema/ulcers; grade 3 - ulcers/exclusively liquid diet; grade 4 feeding is not possible (Table 1).

The $\mathrm{IOH}$ and the $\mathrm{AOH}$ groups were compared based on the outcomes OM and OI. Also, chemotherapy type (considering the toxicity potential to the oral mucosa), gender, age group, radiotherapy in the head and neck region, and time to onset of problems were compared.

Statistical analyses included the chi-square test to compare categorical variables and the Mann-Whitney test to compare the degree of $\mathrm{OM}$ between the two oral health groups (appropriate and inappropriate). The Kaplan-Meier curves were used in the time analysis down to the manifestation of OM and OI. The simple and multiple Cox proportional hazards models was used to estimate hazard ratio (HR), which affords to evaluate probability estimates for the occurrence of these outcomes. These tests were employed to assess the independent association between the variables analyzed (oral health, age, gender, and chemotherapy protocol with high and low OM risk) and the outcomes $\mathrm{OM}$ and $\mathrm{OI}$ investigated. Analyses were carried out using the software SPSS v18.0 with significance level of 0.05 .

All procedures were carried out in accordance with the Declaration of Helsinki (2000). This study was approved by the Ethics Committee of the Hospital de Clínicas de Porto Alegre (HPCA), Porto Alegre, Brazil, authorization number 110055. Custodians of all participants signed an informed consent form.

\section{Results}

Sixty-five patients took part in the present study. Of these, 48 patients presented solid tumors $(24 \mathrm{AOH}$ and $24 \mathrm{IOH})$ and 17 had hematologic tumors ( $8 \mathrm{AOH}$ and $9 \mathrm{IOH})$. Thirty-two individuals were included in the $\mathrm{AOH}$ group (19 males and 13 females), and 33 in the IOH group (17 males and 16 females). In total, 839 dental evaluations were carried out to monitor changes in all patients, OM and OI, from inception to resolution. Of the 33 children with $\mathrm{IOH}$, only 16 were

Table 1. Oral toxicity classification according WHO

\begin{tabular}{|l|c|c|c|}
\hline & \multicolumn{2}{|c|}{ Oral Mucositis } & \multicolumn{2}{|c|}{ Severe Oral Mucositis } \\
\hline Grade 0 & Grade 1 & Grade 2 & Grade 3 \\
\hline No changes & Pain & Erythema 4 & Ulcers \\
\hline & Erythema & Elcers & Exclusive liquid diet \\
\hline
\end{tabular}


given dental treatment previous to chemotherapy.

The protocols that included high OM risk chemotherapy were prescribed to 42 patients (19 patients with $\mathrm{AOH}$ and 23 with $\mathrm{IOH}$ ), while 23 received low $\mathrm{OM}$ risk chemotherapy (13 with $\mathrm{AOH}$ and 10 with $\mathrm{IOH})$. Eleven patients were treated with radiotherapy in the head and neck region (Table 2).

Oral hygiene habits (OHH), family commitment to oral health, and stomatological events prior to the diagnosis of neoplasia were evaluated in the population studied. The results show that only $26.15 \%$ of children brushed their teeth (with the help of an adult when necessary), and that $10.76 \%$ did not brush their teeth or did only once a day. In addition, $46.15 \%$ of patients had never been to the dentist (6 months to 17 years). The patients were sorted for age and existing tooth groups. The age group between 6 months and 6 years corresponds to deciduous dentition, formed by 20 teeth of similar anatomic features. In this stage, parents and custodians are in charge of oral hygiene. The age group between 7 years and 12 years corresponds to mixed dentition, formed by deciduous and permanent teeth. The age group between 13 years and 18 years corresponds to the permanent dentition, which will number 28 teeth in total. The age group between 13 years and 18 years corresponds to the complete permanent dentition, with 32 teeth. Complaints previous to oncologic treatment, showing the precarious character of oral health in this children were: pain was cited by $27.69 \%$ of patients, gingival bleeding by $38.46 \%$, burning sensation in the mouth by $6.15 \%$, and difficulties to swallow by $10.76 \%$ of patients.

Only 12 of the 33 patients of the IOH group finishes dental treatment before chemotherapy. Four patients did not finish dental treatment, and 17 started it without the appropriate oral health status. This was due to the urgency to start chemotherapy in these patients.

The decayed, missing, and filled teeth index (DMFTI) evaluates caries risk in a given population. In order to establish the group in which a patient would be included, only decayed teeth were contemplated, since extracted and filled teeth were considered treated, with no apparent infection. Therefore, the presence of only one decayed tooth

Table 2. Characterization of the population studied.

\begin{tabular}{|c|c|c|c|}
\hline Variables & AOH (n=32) & IOH (n=33) & \multirow{2}{*}{ P } \\
\cline { 2 - 3 } $\begin{array}{c}\text { Age groups } \\
\text { (Dentition stage) }\end{array}$ & $\mathbf{N}(\mathbf{\%})$ & $\mathbf{N}(\mathbf{\%})$ & 0.845 \\
\hline $0-6$ (deciduous) & $18(56.3)$ & $18(54.5)$ & \\
\hline $7-12$ (mixed) & $8(25.0)$ & $7(21.2)$ & \\
\hline $13-18$ (permanent) & $6(18.8)$ & $8(24.2)$ & 0.698 \\
\hline Gender & $13(40.6)$ & $16(48.5)$ & \\
\hline Female & $19(59.4)$ & $17(51.5)$ & \\
\hline Male & & & \\
\hline $\begin{array}{c}\text { Toxic potential of } \\
\text { chemotherapeutic } \\
\text { drugs }\end{array}$ & $19(59.4)$ & $23(69.7)$ & \\
\hline High & $13(40.6)$ & $10(30.3)$ & \\
\hline Low & & & \\
\hline $\begin{array}{c}\text { Chemotherapy }+ \\
\text { Radiotherapy of the } \\
\text { head and neck region }\end{array}$ & & & \\
\hline Yes & $6(18.8)$ & $5(15.2)$ & \\
\hline No & $26(81.3)$ & $28(84.8)$ & \\
\hline Death & $1(2.5 \%)$ & $5(15.6 \%)$ & \\
\hline & & \\
\hline
\end{tabular}

$\mathrm{AOH}=$ appropriate oral health status

$\mathrm{IOH}=$ inappropriate oral health status

$\mathrm{P}<0,05$ already characterized the patient as having $\mathrm{IOH}$.

The visible plaque score (VPS) and the gingival bleeding index (GBI) represent oral hygiene quality and the occurrence of gingivitis, respectively. Gingivitis is one of the most common manifestations of odontogenic infection, besides caries. VPS and GBI were higher in the $\mathrm{IOH}$ patients.

The most common neoplasia observed in the population studied was acute lymphoblastic leukemia (ALL) (20\%), followed by Burkitt's lymphoma (10.8\%), Ewing's sarcoma (9.2\%), and osteosarcoma (7.7\%) (Figure 1).

The frequency of $\mathrm{OM}$ on a patient basis was similar across the population studied, independently of the oral health group $(\mathrm{AOH}$ or $\mathrm{IOH}$ ) (Figure 2). In addition, $\mathrm{IOH}$ patients presented higher incidence and frequency of OI (P 0.003) (Table 3).

In the present study, low-intensity laser therapy (LILT) was used to obtain an analgesic effect and to help the oral mucosa cicatrization, and complemented with chlorhexidine $0.12 \%$ mouthwash were prescribed to prevent secondary contamination of lesions of the oral mucosa, with good clinical response.

While only one $(3.1 \%)$ patient of the $\mathrm{AOH}$ group had more than five OI episodes, eight patients (24.2\%) from the IOH group presented this outcome more than five times during the 6-month follow-up (Table 4).

The data also reveal that $\mathrm{IOH}$ patients were more often diagnosed with $\mathrm{OI}$ than the $\mathrm{AOH}$ group ( $\mathrm{P}$ 0.001), independently of the chemotherapeutic protocol used (Table 4). Eruption cysts and dental and gingival infections were recorded, evaluated, and treated. Two of the oral complications observed in $\mathrm{IOH}$ patients were osteomyelitis and pericoronitis (Figures 3 and 4). The children who received partial dental treatment had higher rate of OI when compared with the children who finished dental treatment before chemotherapy. Incidence of OM was similar in both patient groups (OM has been associated with high risk and low risk chemoterapic protocols).

When patient age was considered, individuals presenting deciduous dentition (6 months to 6 years old) had lower incidence of OM (69.2\%) and OI (15.4\%), while those with permanent dentition (13-18 years old) exhibited higher prevalence of OM (82.4\%) and OI (41.2\%). Patients

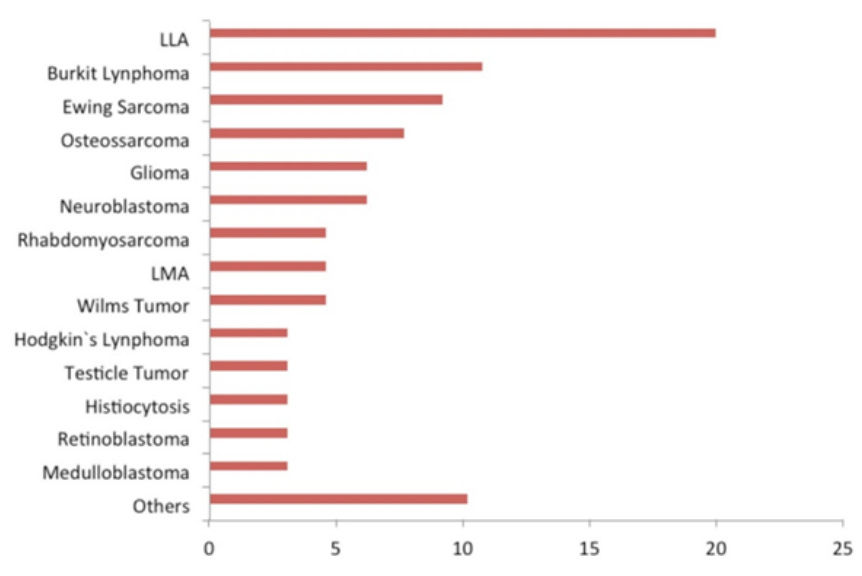

Figure 1. Distribution frequency of patients based on diagnosis of oncological conditions referred to the Pediatric Oncology Service, Teaching Hospital, Federal University of Rio Grande do Sul (HPCA), Porto Alegre, Brazil. 
Table 3. Comparison of OM and OI outcomes in terms of oral health status.

\begin{tabular}{|c|c|c|c|c|}
\hline Outcomes & AOH (n=32) & IOH (n=33) & P & RR (CI 95\%) \\
\hline $\begin{array}{c}\text { Oral mucositis } \\
(\mathrm{OM})\end{array}$ & $\mathbf{n}(\mathbf{\%})$ & $\mathbf{n})$ & & 1.05 \\
\hline Yes & $24(75.0)$ & $26(78.8)$ & & $(0.80-1.37)$ \\
\hline No & $8(25.0)$ & $7(21.2)$ & & 4.85 \\
\hline $\begin{array}{c}\text { Odontogenic } \\
\text { infection (OI) }\end{array}$ & $3(9.4)$ & $15(45.5)$ & & $(1.55-15.15)$ \\
\hline Yes & $29(90.6)$ & $18(54.5)$ & & \\
\hline No & & & & \\
\hline
\end{tabular}

$\mathrm{AOH}=$ appropriate oral health status

$\mathrm{IOH}=$ inappropriate oral health status $* \mathrm{P}<0,05$

Table 4. Comparison of $\mathrm{OM}$ and $\mathrm{IO}$ rates in $\mathrm{AOH}$ and $\mathrm{IOH}$, and potencial drugs toxicity.

\begin{tabular}{|c|c|c|c|c|c|c|}
\hline Outcomes & $\begin{array}{c}\text { AOH } \\
(\mathbf{n = 3 2})\end{array}$ & $\begin{array}{c}\text { IOH } \\
\mathbf{( n = 3 3 )}\end{array}$ & & & \\
\hline & $\begin{array}{c}\text { High risk } \\
(\mathbf{n = 1 9 )}\end{array}$ & $\begin{array}{c}\text { Low risk } \\
(\mathbf{n}=\mathbf{1 3})\end{array}$ & $\mathbf{P}$ & $\begin{array}{c}\text { High risk } \\
(\mathbf{n = 2 3})\end{array}$ & $\begin{array}{c}\text { Low risk } \\
(\mathbf{n = 1 0})\end{array}$ & $\mathbf{P}$ \\
\hline OM & & & $0.001^{*}$ & & & 0.646 \\
\hline Yes & $19(100)$ & $5(38.5)$ & & $19(82.6)$ & $7(70.0)$ & \\
\hline No & $0(0.0)$ & $8(61.5)$ & & $4(17.4)$ & $3(30.0)$ & \\
\hline OM grade & & & 0.958 & & & 0.440 \\
\hline I & $4(27.1)$ & $1(20.0)$ & & $3(15.8)$ & $3(42.9)$ & \\
\hline II & $11(57.9)$ & $3(60.0)$ & & $9(47.4)$ & $3(42.9)$ & \\
\hline III & $1(5.3)$ & $0(0.0)$ & & $2(10.5)$ & $0(0.0)$ & \\
\hline IV & $3(15.8)$ & $1(20.0)$ & & $5(26.3)$ & $1(14.3)$ & \\
\hline OI & & & 0.058 & & & 0.722 \\
\hline Yes & $0(0.0)$ & $3(23.1)$ & & $11(47.8)$ & $4(40.0)$ & \\
\hline No & $19(100)$ & $10(76.9)$ & & $12(52.2)$ & $6(60.0)$ & \\
\hline
\end{tabular}

$\mathrm{AOH}=$ appropriate oral health status

$\mathrm{IOH}=$ inappropriate oral health status

$\mathrm{OM}=$ oral mucositis

$\mathrm{OI}=$ odontogenic infection

$* \mathrm{P}<0,05$

with mixed dentition ( 7 to 12 years old) presented both deciduous and permanent teeth. In this group, incidence of OM was high $(81.8 \%)$, while the prevalence of OI was between the values observed for the other two dentition groups (31.8\%). In terms of gender, we observed that males were more frequently affected $(80.6 \%)$ by OM, while female patients exhibited higher incidence of OI (41.4\%).

When the incidence of OI after chemotherapy of different toxic potentials is analyzed, it became clear that no $\mathrm{AOH}$ patient developed OI when treated with high OM risk chemotherapy. On the other hand, the incidence of OI was $47.8 \%$ in IOH patients who had been treated with the same regimen, which suggests that previous infectious processes are a strong risk factor for the onset of stomatological complications. It was observed that $40 \%$ of the IOH group and $23.1 \%$ of the $\mathrm{AOH}$ group had OI after low OM risk chemotherapy, though with no statistical significance, due to the small sample size (Table 4).

Due to the small number of patients who were given radiotherapy on the head and neck region associated with chemotherapy, the results for this group were not statistically significant. All 11 patients presented solid tumors with prescription of radiotherapy in addition to chemotherapy using low OM risk drugs. The IOH patients (five) presented more complications compared with those with $\mathrm{AOH}$ (six patients). Sequelae associated with radiotherapy on the head and neck region were observed in both groups (xerostomia, radiation caries, changes in tooth germens, and anodontia).

Both $\mathrm{OM}$ and $\mathrm{OI}$ occurred earlier in $\mathrm{IOH}$ patients than in $\mathrm{AOH}$
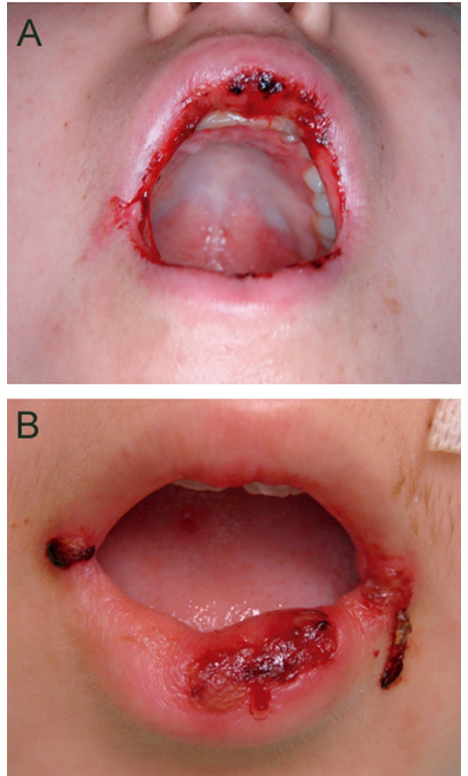

Figure 2. Chemotherapy patients with $\mathrm{OM}$ grade 3. Patient female, 15 years, with osteosarcoma, presented several ulcers on the inside and outise of lips. At this stage, OM patients consume only liquid foods (grade 3 ).
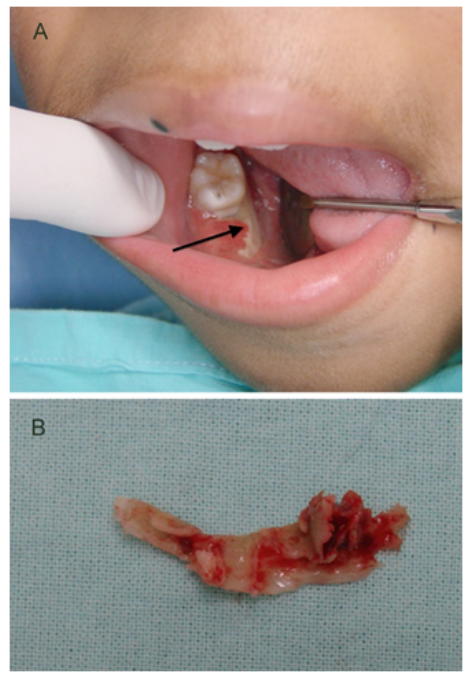

Figure 3. Male patient, 9 years old, developed AML and was admitted to hospital for cancer treatment. (A) Osteomyelitis on the right side of the mandible was diagnosed after tooth extraction (arrow). The patient was treated with systemic antibiotics, daily irrigation with hydrogen peroxide $10 \mathrm{v}$ and sodium iodide $2 \%$, followed by removal of sequestrum (B).

patients, considering the beginning of chemotherapy. This suggests that poor oral health conditions may predispose patients to these complications, especially in the more severe forms of OM and of OI (Figure 5).

Cox regression revealed that the $\mathrm{IOH}$ patients were at a 5.6 times higher risk of acquiring OI than $\mathrm{AOH}$ patients (CI: 95\%; $1.62-19.52$ ). Cox regression for oral health status, gender, and age group showed that the $\mathrm{IOH}$ patients were at a 5.1 higher risk of OI, compared with the AOH group (CI: 95\%; 1.44 - 18.32). In addition, compared to male patients, female patients were at a 2.3 higher risk of acquiring OI, though with no statistically significant difference (CI: 95\%; $0.88-6.36$ ). The same was observed for patients with permanent dentition, with a 1.9 higher risk of OI (CI: 95\%; 0.57 - 6.93) (Figure 5). 


\section{Discussion}

The treatment of cancer in children and adolescents requires appropriate oncologic treatment and management of the likely toxicity reactions emerging thereof. The oral mucosa often is target to these aggressive agents, resulting in OM. Due to the severity of OM, feeding through the oral route is impossible, leading to the interruption of chemotherapy so that the patient may recover and then proceed with the treatment. In addition, $\mathrm{OM}$ is the gateway to microbial agents that may cause sepsis and death [10]. Loeffen et al. (2015) developed a guideline enumerating the themes of the highest clinical demand, and OM was listed under the ten topics most cited by oncologists, nurses, and pediatricians [11].

Few studies have described the aspects associated with the oral health conditions of patients under cancer treatment $[6,7,12,13]$. The patient population included in the present study was formed by children starting at the age of 6 months and young adults at the age of 18 with positive diagnosis of malignant tumors and prescription

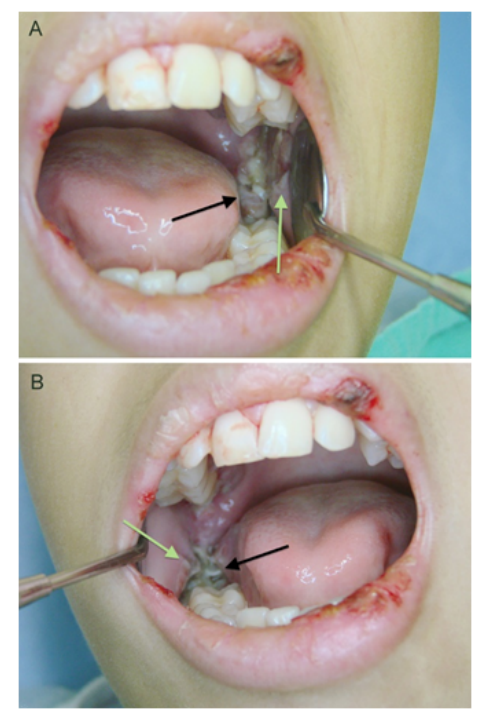

Figure 4. Male patient, 12 years old, with ALL, developed bilateral pericoronitis (black arrows) associated with the eruption of the second lower molars. Edema and trauma ulcers were also observed bilaterally on the mucosa next to tooth in eruption process (green arrows). Apart from OI, the patient presented mucositis grade on lips. OM promotes the dissemination of bacteria present in pericoronaritis. Hospital admission, systemic antibiotic therapy, nutritional support, and local pain and infection management were required. of chemotherapeutic and/or radiotherapeutic treatment. Age group, gender, and treatment prescribed distributed similarly across patients of the $\mathrm{AOH}$ and $\mathrm{IOH}$ groups.

Caries and periodontal disease are infectious conditions that may increase morbidity and mortality in a scenario of neutropenia after chemotherapy, increasing susceptibility to infections. Ideally, odontological treatment has to be carried out before chemotherapy or radiotherapy so as to prevent complications caused by cancer treatment [14].

The oral cavity was prepared filling and extracting teeth, prophylaxis, and topic use of fluoride. The importance of oral care and tooth brushing was highlighted in all appointments. Compliance with directions and oral care were not correctly observed in some cases, which may have caused the infections recorded.

The role of parents in the promotion and maintenance of oral health has been discussed in the literature [15]. Oral care should be taught and incentivized, but children lack the motor capabilities to brush teeth correctly, pointing to the need for supervision by parents or custodians. Eating habits also influence oral health and should be included in this education process [16].

A study that evaluated the oral health conditions in pre-school children revealed high prevalence and severity of oral health problems that could be prevented and controlled based on educational measures [17]. In the present study, the instructions presented to parents and custodians listing oral healthcare and post-chemotherapy recovery instructions was effective to reduce the most severe OI.

It is known that OM the stomatological complication most often associated with chemotherapy, being reported in as many as $40 \%$ of adult cancer patients. What influences the progression of $\mathrm{OM}$ is not the type of tumor, but the chemotherapeutic protocol that includes drugs with known stomatotoxicity. OI are associated with oral health conditions during chemotherapy. The worse the oral health condition, the higher the risk of odontogenic infections.

Yet, in the present study, OM was observed in over $70 \%$ of the pediatric cancer patients included. It is not possible to confirm that these children were more sensitive to chemotherapy, since it should be remembered that this difference in prevalence observed herein is also affected by medical drugs prescribed in pediatric protocols that often are more toxic to this age group than to adults. Cheng et al.
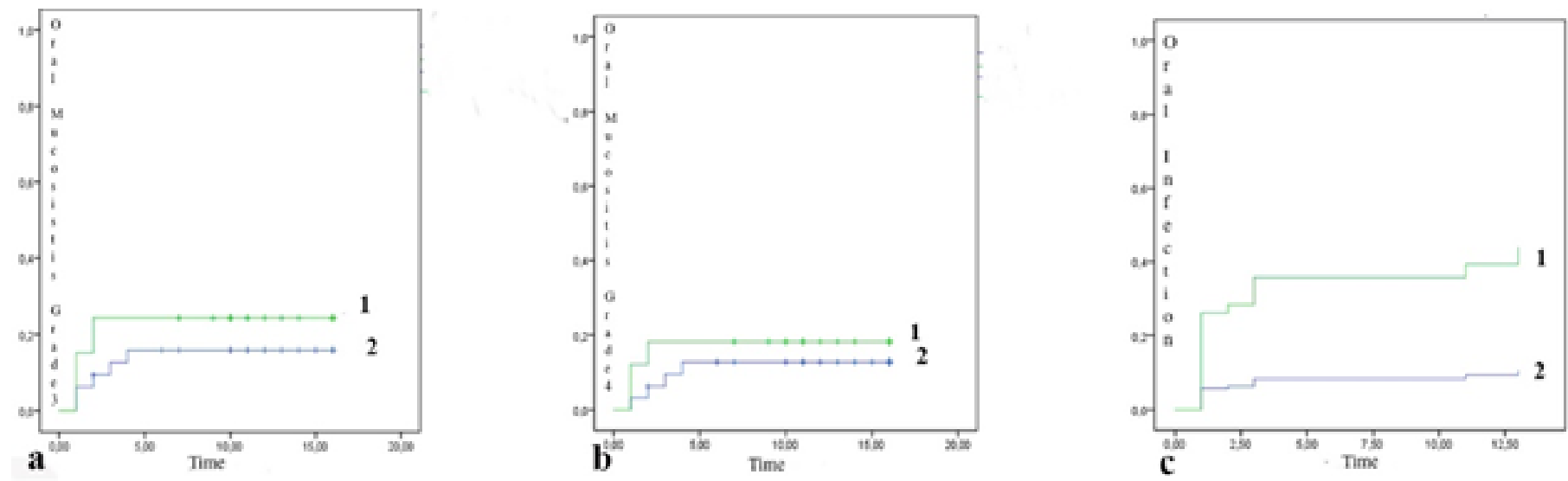

Figure 5. Kaplan-Meier curves used in the time analysis from the beginning of chemotherapy, onset of OM and of OI. a) OM grade 3 manifested earlier in the IOH group (1) compared to the $\mathrm{AOH}$ group (2); b) OM grade 4 manifested earlier in the IOH group (1) compared to the AOH group (2); c) OI occurred earlier in the IOH group (1) compared with the AOH group (2). The $\mathrm{X}$ axis indicates time, while the $\mathrm{Y}$ axis indicates the outcomes OM and OI. 
(2004) reported OM in approximately $52 \%-80 \%$ of children under chemotherapy, and argue that the high prevalence of the condition in children and adolescents may be ascribed to the rate of cell division of the basal layer in a neutropenia scenario. They also claim that high susceptibility to OM may be linked with the fact that most pediatric tumors are hematological in character, and that the therapeutic protocols used in these cases are more toxic to the mucosa when compared with the drugs used to treat solid tumors, which are more prevalent in adults $[8,18]$. Fernández et al. (2012) reported that the severity of OM correlates positively with age, when younger patients present more severe lesions [19].

Here, the prevalence of OM was similar across the two groups $\mathrm{IOH}$ and $\mathrm{AOH}$. When the occurrence of $\mathrm{OM}$ was analyzed in light of the toxicity of the drugs prescribed, we observed increased risk for patients that received high-risk chemotherapy in both the $\mathrm{AOH}$ and IOH groups. This finding confirmed what was expected; however, the analysis of the time to emergence of OM shows that the condition appeared more precociously in the $\mathrm{IOH}$ group, suggesting that oral health condition may precipitate this outcome.

A recent systematic review based on findings about OM prevention in pediatric cancer patients suggests that oral health protocols are efficient and accessible. Few studies have looked into the effects of other treatments prescribed to pediatric patients, namely mouthwashes and applications of sucralfate, glutamine, chlorhexidine digluconate, and laser therapy. More research is needed to confirm previous results [20].

A randomized clinical study with adults and children showed that the association of laser therapy and oral hygiene directions reduced the span of OM induced by chemotherapy and radiotherapy [21,22]. In a meta-analysis, Sapna Oberoi et al. demonstrated that prophylaxis based on LILT reduces the risk and duration of severe OM. The approach also reduces the risk of intense pain that requires opioids and unplanned interruptions of radiotherapy sessions [23].

Odontogenic infections such as gingivitis were present in $30 \%$ of the $\mathrm{IOH}$ group, while in the $\mathrm{AOH}$ group the infections associated with gingivitis were diagnosed in $9.4 \%$ of subjects. Also, in the IOH group these infections emerged before, compared with the AOH group. The relative risk of the IOH group was at to present this complication was 4.85 , confirming the strong association between oral health and this finding. It was expected that previous treatment of the oral cavity would place both groups on equal foot in terms of oral health and that, as a result, OM prevalence would be similar. This increased risk may be explained in light of the fact dental treatment of some patients of this group had to be interrupted due to the urgency to start cancer treatment. The educational and awareness measures transmitted to patients likewise failed to reduce the risk of OI.

Early dental evaluation coupled with dental treatment and the adoption of an oral health protocol remain the determining factors in the prevention of stomatological complications during cancer treatment. Clinical follow-up of these patients based on frequent evaluations was important in the effort to start treatment of OM early and thus prevent worsening of the condition.

The limitations of this study include (i) the small number of patients, since the incidence of cancer in children is low; (ii) the inclusion of all patients with diagnosis of any neoplasia (whether solid or hematologic tumors), (iii) the fact that the classification of oral health as appropriate or inappropriate was adopted only in the beginning of the treatment; (iv) and the impossibility to finish dental treatment before chemotherapy in $\mathrm{IOH}$ patients.

\section{Conclusion}

The factors associated with higher prevalence of OM were gender (male), age group (eruption of permanent dentition), and use of drugs with confirmed toxic potential to trigger OM. Despite the incidence rates of $\mathrm{OM}$ in $\mathrm{AOH}$ and $\mathrm{IOH}$ being similar, the condition affected $\mathrm{IOH}$ patients earlier. Concerning OI, these were more prevalent in $\mathrm{IOH}$ women at the age of permanent dentition eruption. However, we believe that, despite these results, interventions were efficacious to prevent severe stomatological complications. Few studies have analyzed dental interventions in pediatric cancer patients. The present study shed new light on the profile and needs expressed by dental patients, producing essential information to prepare new treatment protocols to this population.

\section{Authorship}

Isabel Nemoto Vergara Sasada: conception, design, acquisition of data, analysis and interpretation data, drafting the article, final approval of the version to be published.

Maria Cristina Munerato: conception, design, analysis and interpretation data, drafting the article, revising it critically for important intellectual content, final approval of the version to be published.

Lauro José Gregianin: conception, design, analysis and interpretation data, drafting the article, revising it critically for important intellectual content, final approval of the version to be published, agreement to act as guarantor of the work.

\section{Acknowledgments}

Our acknowledgments to Vania Naomi Hirakata for her contribution to do the statistical analysis.

\section{References}

1. Instituto Nacional de Câncer José Alencar Gomes da Silva Estimativa 2012: incidência de câncer no Brasil. (National Cancer Institute José Alencar Gomes da Silva. Estimate 2012: cancer incidence in Brazil). Rio de Janeiro.

2. Bailey LC, Reilly AF, Rheingold SR (2009) Infections in pediatric patients with hematologic malignancies. Semin Hematol 46: 313-324. [Crossref]

3. Epstein JB, Schubert MM (2003) Oropharyngeal mucositis in cancer therapy. Review of pathogenesis, diagnosis, and management. Oncology (Williston Park) 17: 17671779. [Crossref]

4. National Cancer Institute (2013) The National Institutes of Health. Oral Mucositis.

5. Chow AW (2013) Epidemiology, pathogenesis and clinical manifestations of odontogenic infections.

6. World Health Organization (2003) Oral Health Report.

7. Ministry of Health (2010) National Oral Health Survey, Note for the press. Brazil.

8. Albuquerque RUA, Morais VL, Sobral APV (2007) Protocolo de Atendimento Odontológico a Pacientes Oncológicos Pediátricos - Revisã de Literatura. (Dental Care Protocol to Pediatric Oncology Patients - Literature Review.) Rev odontol UNESP 36: 275-280.

9. Cheng KKF, Chang AM, Yuen MP (2004) Prevention of oral mucositis in paediatric patients treated with chemotherapy: a randomised crossover trial comparing two protocols of oral care. Eur J Cancer 40: 1208-1216. [Crossref]

10. Keefe DM, Schubert MM, Elting LS, Sonis ST, Epstein JB, et al. (2007) Updated clinical practice guidelines for the prevention and treatment of mucositis. Cancer 109: 820-831. [Crossref]

11. Loeffen EA, Mulder RL, Kremer LC, Michiels EM, Abbink FC, et al. (2015) Development of clinical practice guidelines for supportive care in childhood cancer- 
prioritization of topics using a Delphi approach. Support Care Cancer 23: 1987-1995. [Crossref]

12. Azher U, Shiggaon N (2013) Oral health status of children with acute lymphoblastic leukemia undergoing chemotherapy. Indian J Dent Res 24: 523. [Crossref]

13. Pels E, Mielnik-Blaszczak M (2012) Oral hygiene in children suffering from acute lymphoblastic leukemia living in rural and urban regions. Ann Agric Environ Med 19: 529-533. [Crossref]

14. Fawad F, Utreja A, Correa FOB, Al-Askar M, Hudieb M, et al. (2016) Oral health status in children with acute lymphoblastic leukemia. Crit Rev in Oncol Hematol 83: 303-309. [Crossref]

15. Alves UM, Volschan BCG, Haas NA (2004) Educação em Saúde Bucal. (Education in Oral Health.) Pesq. Bras Odontop Clín Integr João Pessoa 4: 47-51.

16. Oliveira Album, Botta AC, Rosell FL (2010) Promoção de Saúde Bucal em Bebês. (Oral Health Promotion in babies). Revista de Odontologia da Universidade de São Paulo 22: 247-253.

17. Almeida TF, Cangussu MCT, Chaves SCL, Silva DIA de C, Santos SC (2009) Condições de Saúde Bucal de crianças na faixa etária pré-escolar, residentes em áreas de abrangência do Programa Saúde da Família em Salvador, Bahia, Brasil. (Oral health conditions of children in preschool age, living in areas covered by the Family Health Program in Salvador, Bahia, Brazil). Rev Bras de Saúde Mater Infant 9: 247-252.
18. Instituto Nacional do Câncer, Ministério da Saúde (2011) Diagnóstico Precoce do Câncer na Criança e Adolescente. (National Cancer Institute, Ministry of Health. Early Diagnosis of Cancer in Children and Adolescents.) Rio de Janeiro.

19. Fernández AH, Sánchez REO, Merino MCC, Fuente FA, Fernando IH , et al. (2012) Influence of oral health on mucositis in patients undergoing hematopoietic progenitor cell transplantation (HPCT). Med Oral Patol Oral Cir Bucal 17: 94-101. [Crossref]

20. Qutob AF, Gue S, Revesz T, Logan RM, Keefe D (2013) Prevention of oral mucositis in children receiving cancer therapy: a systematic review and evidence-based analysis. Oral Oncol 49: 102-107. [Crossref]

21. Kuhn A, Porto FA, Miraglia P, Brunetto AL (2009) Low-level infrared laser therapy in chemotherapy-induced oral mucositis: a randomized placebo-controlled trial in children. J Pediatr Hematol Oncol 31: 33-37. [Crossref]

22. Eduardo Fde P, Bezinelli LM, de Carvalho DL, Lopes RM, Fernandes JF, et al. (2015) Oral mucositis in pediatric patients undergoing hematopoietic stem cell transplantation: Clinical outcomes in a context of specialized oral care using low-level laser therapy. Pediatr Transplant 19: 316-25. [Crossref]

23. Oberoi S, Zamperlini-Netto G, Beyene J, Treister NS, Sung L (2014) Effect of prophylactic low level laser therapy on oral mucositis: a systematic review and metaanalysis. PLoS One 9: 107418. [Crossref]

Copyright: $\odot 2016$ Sasada INV. This is an open-access article distributed under the terms of the Creative Commons Attribution License, which permits unrestricted use, distribution, and reproduction in any medium, provided the original author and source are credited. 